\title{
Book Sales and Readership Statistics: Ukrainian Trends 2021
}

\author{
K. Astaptseva
}

\begin{abstract}
The research study explores the phenomenon of consumer book buying behavior in COVID-19. The study focuses on Ukrainian reader's taste. We applied a questionnaire and obtained 100 responses. How much do people spend on books per month? What types of books are they buying? What genres are they selecting? Consumer insights play main role in a publisher's strategies, from acquisitions to pricing and marketing campaigns. The results here indicate book buying habits of Ukrainian readers who participated in the survey in Kyiv "Bukva" bookshop in February 2021.
\end{abstract}

Key words - Best sellers in books, consumer behavior, readership statistics, Ukrainian bookstore "Bukva".

\section{INTRODUCTION}

In Ukraine books remain an important part of consumers' daily lives. The current bookstore landscape now includes Yakaboo, Ye Bookstore, BUKVA as well as smaller chains and independent bookstores, such as Bookovka.ua and other.

How much do people spend on books per month? What types of books are they buying? What genres are they selecting? Consumer insights play main role in a publisher's strategies, from acquisitions to pricing and marketing campaigns. We would like to share highlights from book consumer demographics \& buying behaviors review, compiled on the basis of the bookstore chain "Bukva" ["The Letter"] sales in February 2021.

"Bukva" ["The Letter"] is a leader in the distribution market of cultural goods with a unique heritage and the legendary history [1].

"Bukva" - is a chain of Ukrainian modern bookstores (more than 22 bookstores in 11 cities - Kyiv, Vinnicya, Zaporizhzhya, Odesa, Poltava, Rivne, Sumy, Ternopil, Kherson, Khmelnitsky, Chernivtsi). "Bukva" offers customers a wide range of books. Customers can buy classical and contemporary fiction, business literature and popular science editions, children's books for any age category, stationery, bright leaflets, and games. In "Bukva" customers will always find actual book novelties of various genres and trends, attractive new discounts, and interesting special offers [1].

BUKVA.ua - one of the largest online bookstores in Ukraine. For regular customers operates discount program. Delivery of orders is carried out throughout Ukraine and abroad [1].

\section{RESEARCH BACKGROUND}

Statistics about book publishing in Ukraine are maintained by the state research institution, the Ivan Fedorov Book Chamber of Ukraine [2]. According to its data, the number of new titles and reprints published during the 2021 can be characterized as follows (see Table I).

TABLE I: The Number of NEW TITLES AND REPRINTS Published DURING THE 2021

\begin{tabular}{ccc}
\hline & \multicolumn{2}{c}{2021} \\
\cline { 2 - 3 } $\begin{array}{c}\text { Types of } \\
\text { publications }\end{array}$ & $\begin{array}{c}\text { Number of titles, } \\
\text { publishing units }\end{array}$ & $\begin{array}{c}\text { Number of } \\
\text { copies, } \\
\text { thousand }\end{array}$ \\
\hline Books and brochures & 2692 & 1679.4 \\
Dissertation & 1360 & 136.0 \\
Abstracts & 106 & 270.0 \\
Art books &
\end{tabular}

During the last ten years in Ukraine were done only 3 massive research surveys:

A. Publishing and bookselling in Ukraine: Facts, Trends, Recommendations. Results of a survey carried out in 2012

It presents a summary of the study of the Ukrainian book sector and its main findings and conclusions, as well as two expert opinions on it. This is the first time that a study of book publishing and book distribution in Ukraine has been carried out on such a scale. The study is based on years of systematic collection of information by the Ukrainian Association of Book Publishers and Book Sellers (UABB), while the report was commissioned by the Publishers Forum (Lviv) as part of the Book Platform project. The report provides information about the state of the Ukrainian publishing sector from 2002 until 2012 (The information for 2012 is not presented in all sections, as it had not been processed at the time of the Report's compilation).

Authors:

- Survey methodology, data collection and report by Oleksandr Afonin;

- Comments on the book sector survey by Rüdiger Wischenbart;

- The book sector survey report - a summary by Myroslava Prykhoda;

- Conclusions and Recommendations by Oleksandra Koval [3].

\section{B. Ukrainian Reading and Publishing Data 2018}

The all-Ukrainian big project aimed to represent a state of the modern publishing market, readers' preferences and, by 
that, give the objective information that can influence forming the policy of both the cultural institutions and editorial office portfolios of publishers, as well as give a material to present the Ukrainian market abroad in an efficient visual format - infographic presentation. With the research as a basis, where reader's preferences and the state of the market 2018 was revealed, to create conditions for development of the publishing sphere against the background of the objective and actual data.

Organizers:

- Gutenbergz, a digital publishing house, which unites the story and the interactivity, and creates wonderful stories for mobile devices, cross-platform solutions for the multi-media content, namely for the learning materials in a comfortable and effective format.

- Cultural and publishing project Chytomo, media about the book in all its forms and about reading as a springboard for self-education and realization.

- Razumkov Centre, the leading non-government analytical center of Ukraine that conducts research on the state policy, was established in 1994 [4].

\section{Publishing in Ukraine: a review of the sector. Final report 2020}

This research was commissioned by the British Council and authored by Emma Shercliff. Emma is a publishing consultant with over 20 years of experience in the industry. She has worked for publishing houses in Paris, Melbourne, Abuja, and London, and for the British Council in Nigeria and Iran.

The results of this study should prove useful to both foreign partners as well as Ukrainian institutions, publishers, booksellers, authors, and other actors in this segment. This independent, external view of the field provides a muchneeded challenge to entrenched perspectives, with solutions proffered arising from a welcome reconsideration of the challenges facing the sector to the benefit of both stand-alone projects and the broader publishing industry.

Of particular note is the attention given in the study to the undergraduate community and instructors at industry-related university programs - the wellspring of emerging publishers, managers, agents, mediators, authors, and literary critics soon poised to join the market [5].

Also, for 2018-2020 years there were published some statistic rewievs about Ukrainian book business:

- Gerden, E. (2018). Retail Pressures: Ukraine's OpenAir Bookselling Marketplaces Appear Headed for Closure [6].

- Stepurin, I. (2019). Book market of Ukraine [7].

- Gerden, E. (2020). Ukraine Book Business After Lockdowns: An 'Almost Complete Collapse [8].

- Rybak, V. (2020). Reading in Ukrainian: The Resurgence of the Ukrainian Book Industry [9].

\section{Methodology}

The method design consisted of applying a paper form, with eleven statements, based on a 5-point Likert scale (with the options completely disagree $=1$, disagree $=2$, neutral $=$ 3 , agree $=4$, and completely agree $=5$ ). The form was applied between January 2021 to February 2021 for customers who visited bookshop "Bukva" ["The Letter"], address location: Ukraine, Kyiv, Arsenalna metro station, Slavy Square [Glory Square], 1, Underpass Arsenal, work time: from 10-00 till 2000 . The author's questionnaire statements were constructed experimentally and previously tested to detect the perception that the professionals had of the readers about the reading of the visualization. In the pilot study, the following questions were adopted:

- Your gender;

- Your age;

- Your education;

- Which book genre you most often purchase;

- Do you purchase books at online or offline bookstores;

- How often do you purchase a books;

- How often do you visit your local library;

- Do you prefer paper books or e-books;

- How much money are you ready to spend on books;

- Name the life-changing books that shaped your thinking;

- Name the books you've recently read.

In total, the questionnaire was applied to 134 people, reaching a return rate of $25,37 \%$, with 34 responses. Ten were excluded due to incomplete questionnaire. In the end, we worked with 100 responses. In addition to statements and identity, we also asked about age, profession, and educational background. The items on age and training will be used to analyze the results to test the hypotheses. The average age of those who participated in the questionnaire is 40,8 years, 32 men and 68 women.

The population of the survey consists of persons aged from 13 to 71 who permanently live in Kyiv. Table II shows a part (without including answers on question "Which book genre you most often purchase") of respondents' answers recorded on a questionnaire form.

\section{RESUlTS AND DisCUSSION}

Demographic Information about respondents can be seen on the Fig. 1, and 3. On a gender basis, women are the more avid book readers, per the study, being $36 \%$ more likely than men to have read a book in the prior 6 months (68\% vs. $32 \%)$.

Separately, the results show that the more highly educated the respondents, the more likely they are to have read a book, with a similar positive correlation evident with income. Due to results of questionnaire, $65 \%$ respondents have master's degree, $22 \%$ have bachelor degree, $12 \%$ have secondary education, $1 \%$ has a $\mathrm{PhD}$ degree.

Usually education depends on age: all respondents older than 23 years have master's degree. Over $80 \%$ of respondents have at least some college educations, if not a degree.

As we can see from the table, much book readers are 2040-year-old (52,6\%), less -13-20 year old (24,7\%), 40-60 year old $(17 \%)$ and the smallest group consists of $60-71$ year old respondents $(5,2 \%)$. 
TABLE II: RESPONDENTS' ANSWERS RECORDED ON A QUESTIONNAIRE FORM

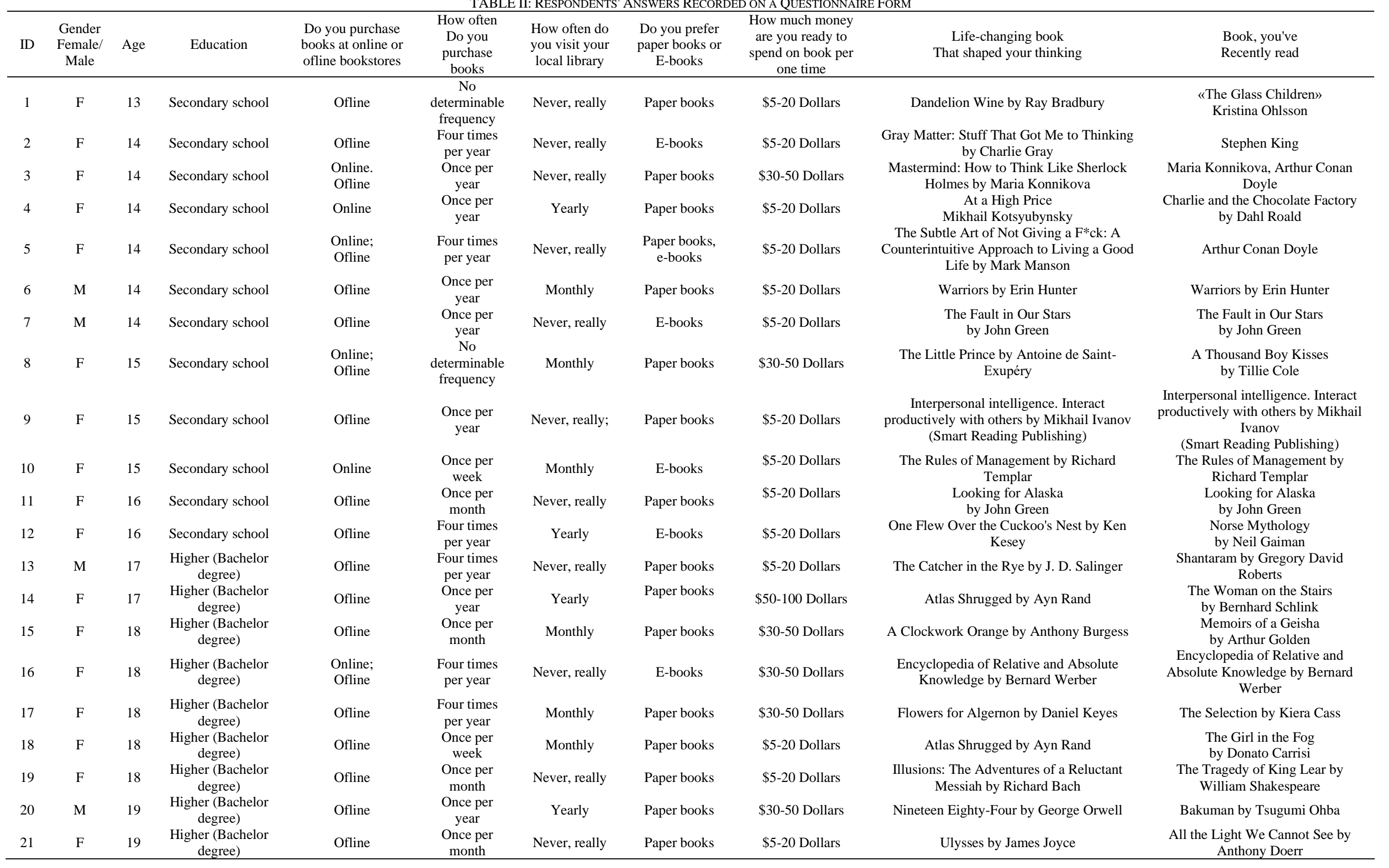




\begin{tabular}{|c|c|c|c|c|c|c|c|c|c|c|}
\hline ID & $\begin{array}{l}\text { Gender } \\
\text { Female/ } \\
\text { Male }\end{array}$ & Age & Education & $\begin{array}{l}\text { Do you purchase } \\
\text { books at online or } \\
\text { ofline bookstores }\end{array}$ & $\begin{array}{l}\text { How often } \\
\text { Do you } \\
\text { purchase } \\
\text { books }\end{array}$ & $\begin{array}{l}\text { How often do } \\
\text { you visit your } \\
\text { local library }\end{array}$ & $\begin{array}{l}\text { Do you prefer } \\
\text { paper books or } \\
\text { E-books }\end{array}$ & $\begin{array}{l}\text { How much money } \\
\text { are you ready to } \\
\text { spend on book per } \\
\text { one time }\end{array}$ & $\begin{array}{l}\text { Life-changing book } \\
\text { That shaped your thinking }\end{array}$ & $\begin{array}{l}\text { Book, you've } \\
\text { Recently read }\end{array}$ \\
\hline 22 & $\mathrm{~F}$ & 19 & $\begin{array}{l}\text { Higher (Bachelor } \\
\text { degree) }\end{array}$ & Ofline & $\begin{array}{l}\text { Once per } \\
\text { month }\end{array}$ & Never, really & Paper books & \$5-20 Dollars & $\begin{array}{l}\text { Big Magic: Creative Living Beyond Fear by } \\
\text { Elizabeth Gilbert }\end{array}$ & Lolita by Vladimir Nabokov \\
\hline 23 & $\mathrm{~F}$ & 19 & $\begin{array}{l}\text { Higher (Bachelor } \\
\text { degree) }\end{array}$ & Ofline & $\begin{array}{l}\text { Once per } \\
\text { week }\end{array}$ & Monthly & $\begin{array}{l}\text { Paper books, } \\
\text { e-books }\end{array}$ & \$50-100 Dollars & $\begin{array}{c}\text { In simple words. How to understand your } \\
\text { emotions by Mark Livin; Shantaram by } \\
\text { Gregory David Roberts; Pollyanna by } \\
\text { Eleanor H. Porter, }\end{array}$ & $\begin{array}{c}\text { A Little Life } \\
\text { by Hanya Yanagihara }\end{array}$ \\
\hline 24 & $\mathrm{~F}$ & 19 & $\begin{array}{l}\text { Higher (Bachelor } \\
\text { degree) }\end{array}$ & Ofline & $\begin{array}{l}\text { Once per } \\
\text { year }\end{array}$ & Never, really & Paper books & \$50-100 Dollars & $\begin{array}{l}\text { Emotional Intelligence: Why It Can Matter } \\
\text { More Than IQ by Daniel Goleman }\end{array}$ & $\begin{array}{l}\text { The Man Who Laughs by Victor } \\
\text { Hugo }\end{array}$ \\
\hline 25 & $\mathrm{~F}$ & 20 & $\begin{array}{l}\text { Higher (Bachelor } \\
\text { degree) }\end{array}$ & $\begin{array}{l}\text { Online; } \\
\text { Ofline }\end{array}$ & $\begin{array}{l}\text { Once per } \\
\text { month }\end{array}$ & Never, really & Paper books & \$50-100 Dollars & $\begin{array}{c}\text { Consciousness and civilization by Merab } \\
\text { Mamardashvili }\end{array}$ & Know Thyself by Vladimir Bibikhin \\
\hline 26 & $\mathrm{~F}$ & 20 & $\begin{array}{l}\text { Higher (Bachelor } \\
\text { degree) }\end{array}$ & Ofline & $\begin{array}{l}\text { Four times } \\
\text { per year }\end{array}$ & Yearly & Paper books & $\$ 30-50$ Dollars & $\begin{array}{l}\text { The Little Prince by Antoine de Saint- } \\
\text { Exupéry. }\end{array}$ & $\begin{array}{l}\text { How to Win Friends \& Influence } \\
\text { People by Dale Carnegie }\end{array}$ \\
\hline 27 & $\mathrm{~F}$ & 20 & $\begin{array}{l}\text { Higher (Bachelor } \\
\text { degree) }\end{array}$ & Ofline & $\begin{array}{l}\text { Once per } \\
\text { month }\end{array}$ & Never, really & Paper books & $\$ 30-50$ Dollars & $\begin{array}{l}\text { The Man Who Mistook His Wife for a Hat } \\
\text { and Other Clinical Tales by Oliver Sacks }\end{array}$ & $\begin{array}{l}\text { Gone with the Wind } \\
\text { by Margaret Mitchell }\end{array}$ \\
\hline 28 & M & 20 & $\begin{array}{l}\text { Higher (Bachelor } \\
\text { degree) }\end{array}$ & Online & $\begin{array}{l}\text { Four times } \\
\text { per year }\end{array}$ & Never, really & Paper books & \$50-100 Dollars & $\begin{array}{l}45 \text { tattoos personality. The rules of my life } \\
\text { by Maxim Batyrev }\end{array}$ & $\begin{array}{l}\text { How to Be the Parent You Always } \\
\text { Wanted to Be by Adele Faber }\end{array}$ \\
\hline 29 & M & 20 & $\begin{array}{l}\text { Higher (Bachelor } \\
\text { degree) }\end{array}$ & Ofline & $\begin{array}{l}\text { Four times } \\
\text { per year }\end{array}$ & Yearly & Paper books & $\$ 30-50$ Dollars & $\begin{array}{c}\text { Caligula and Three Other Plays by Albert } \\
\text { Camus }\end{array}$ & $\begin{array}{l}\text { The Myth of Sisyphus by Albert } \\
\text { Camus }\end{array}$ \\
\hline 30 & $\mathrm{~F}$ & 21 & $\begin{array}{l}\text { Higher (Bachelor } \\
\text { degree) }\end{array}$ & Ofline & $\begin{array}{l}\text { Once per } \\
\text { month }\end{array}$ & Monthly & Paper books & \$50-100 Dollars & $\begin{array}{l}\text { We Are Our Brains: A Neurobiography of } \\
\text { the Brain, from the Womb to Alzheimer's by } \\
\text { Dick Swaab }\end{array}$ & $\begin{array}{c}\text { The Fault in Our Stars by John } \\
\text { Green }\end{array}$ \\
\hline 31 & $\mathrm{~F}$ & 21 & $\begin{array}{l}\text { Higher (Bachelor } \\
\text { degree) }\end{array}$ & Ofline & $\begin{array}{l}\text { Once per } \\
\text { month }\end{array}$ & Never, really & Paper books & $\$ 30-50$ Dollars & Me Before You by Jojo Moyes & $\begin{array}{c}\text { The Fault in Our Stars by John } \\
\text { Green }\end{array}$ \\
\hline 32 & $\mathrm{~F}$ & 21 & $\begin{array}{l}\text { Higher (Bachelor } \\
\text { degree) }\end{array}$ & Ofline & $\begin{array}{l}\text { Once per } \\
\text { month }\end{array}$ & Never, really & Paper books & \$50-100 Dollars & $\begin{array}{l}\text { The Effective Executive: The Definitive } \\
\text { Guide to Getting the Right Things Done by } \\
\text { Peter Drucker }\end{array}$ & $\begin{array}{l}\text { The Effective Executive: The } \\
\text { Definitive Guide to Getting the } \\
\text { Right Things Done by Peter Drucker }\end{array}$ \\
\hline 33 & $\mathrm{~F}$ & 21 & $\begin{array}{l}\text { Higher (Bachelor } \\
\text { degree) }\end{array}$ & Ofline & $\begin{array}{l}\text { Once per } \\
\text { month }\end{array}$ & Never, really & Paper books & \$50-100 Dollars & Inferno by Dan Brown & $\begin{array}{c}\text { In bed with your husband. Notes of } \\
\text { the mistress. Wives must read! by } \\
\text { Nika Nabokova }\end{array}$ \\
\hline 34 & $\mathrm{~F}$ & 22 & $\begin{array}{l}\text { Higher (Bachelor } \\
\text { degree) }\end{array}$ & Ofline & $\begin{array}{l}\text { Once per } \\
\text { month }\end{array}$ & Never, really & Paper books & $\$ 30-50$ Dollars & The Catcher in the Rye by J. D. Salinger & A Woman Like Her by Marc Levy \\
\hline 35 & $\mathrm{~F}$ & 23 & $\begin{array}{l}\text { Full higher } \\
\text { education }\end{array}$ & Ofline & $\begin{array}{l}\text { Once per } \\
\text { month }\end{array}$ & Never, really & Paper books & $\$ 30-50$ Dollars & $\begin{array}{c}\text { Breaking The Habit of Being Yourself: How } \\
\text { to Lose Your Mind and Create a New One } \\
\text { by Joe Dr. Dispenza }\end{array}$ & $\begin{array}{l}\text { Rich Dad Poor Dad by Robert } \\
\text { Kiyosaki }\end{array}$ \\
\hline 36 & M & 23 & $\begin{array}{l}\text { Full higher } \\
\text { education }\end{array}$ & Ofline & $\begin{array}{l}\text { Once per } \\
\text { year }\end{array}$ & Yearly & Paper books & $\$ 30-50$ Dollars & The Bible & $\begin{array}{l}\text { All Quiet on the Western Front by } \\
\text { Erich Maria Remarque }\end{array}$ \\
\hline 37 & M & 23 & $\begin{array}{l}\text { Full higher } \\
\text { education }\end{array}$ & Online & $\begin{array}{l}\text { Once per } \\
\text { month }\end{array}$ & Monthly & Paper books & \$5-20 Dollars & $\begin{array}{l}\text { How to Win Friends and Influence People by } \\
\text { Dale Carnegie }\end{array}$ & $\begin{array}{l}\text { The Air Seller by Alexander } \\
\text { Belayev }\end{array}$ \\
\hline 38 & $\mathrm{~F}$ & 24 & $\begin{array}{l}\text { Full higher } \\
\text { education }\end{array}$ & Ofline & $\begin{array}{l}\text { Once per } \\
\text { month }\end{array}$ & Never, really & $\begin{array}{l}\text { Paper books, } \\
\text { e-books }\end{array}$ & $\$ 30-50$ Dollars & $\begin{array}{l}\text { At the Mountains of Madness by H. P. } \\
\text { Lovecraft }\end{array}$ & The Passengers by John Marrs \\
\hline 39 & $\mathrm{~F}$ & 24 & $\begin{array}{l}\text { Full higher } \\
\text { education }\end{array}$ & Ofline & $\begin{array}{l}\text { Once per } \\
\text { year }\end{array}$ & Never, really & Paper books & \$5-20 Dollars & $\begin{array}{l}\text { Do Androids Dream of Electric Sheep? by } \\
\text { Philip K. Dick }\end{array}$ & $\begin{array}{c}\text { Eight White Nights: A Novel by } \\
\text { André Aciman }\end{array}$ \\
\hline 40 & M & 24 & $\begin{array}{l}\text { Full higher } \\
\text { education }\end{array}$ & $\begin{array}{l}\text { Online; } \\
\text { Ofline }\end{array}$ & $\begin{array}{l}\text { Once per } \\
\text { year }\end{array}$ & Never, really & Paper books & $\$ 30-50$ Dollars & Emotional Intelligence by Daniel Goleman & $\begin{array}{l}\text { Pitch Anything: An Innovative } \\
\text { Method for Presenting, Persuading, } \\
\text { and Winning the Deal by Oren Klaff } \\
\text { Three Cups of Tea: One Man's }\end{array}$ \\
\hline 41 & M & 24 & $\begin{array}{l}\text { Full higher } \\
\text { education }\end{array}$ & Ofline & $\begin{array}{l}\text { Once per } \\
\text { year }\end{array}$ & Yearly & $\begin{array}{l}\text { Paper books, } \\
\text { e-books }\end{array}$ & $\$ 30-50$ Dollars & $\begin{array}{l}\text { For Whom the Bell Tolls } \\
\text { by Ernest Hemingway }\end{array}$ & $\begin{array}{c}\text { Mission to Promote Peace ... One } \\
\text { School at a Time } \\
\text { by Greg Mortenson and David } \\
\text { Oliver Relin }\end{array}$ \\
\hline
\end{tabular}




\begin{tabular}{|c|c|c|c|c|c|c|c|c|c|c|}
\hline ID & $\begin{array}{l}\text { Gender } \\
\text { Female/ } \\
\text { Male }\end{array}$ & Age & Education & $\begin{array}{l}\text { Do you purchase } \\
\text { books at online or } \\
\text { ofline bookstores }\end{array}$ & $\begin{array}{l}\text { How often } \\
\text { Do you } \\
\text { purchase } \\
\text { books }\end{array}$ & $\begin{array}{l}\text { How often do } \\
\text { you visit your } \\
\text { local library }\end{array}$ & $\begin{array}{l}\text { Do you prefer } \\
\text { paper books or } \\
\text { E-books }\end{array}$ & $\begin{array}{l}\text { How much money } \\
\text { are you ready to } \\
\text { spend on book per } \\
\text { one time }\end{array}$ & $\begin{array}{l}\text { Life-changing book } \\
\text { That shaped your thinking }\end{array}$ & $\begin{array}{l}\text { Book, you've } \\
\text { Recently read }\end{array}$ \\
\hline 42 & $\mathrm{~F}$ & 25 & $\begin{array}{l}\text { Full higher } \\
\text { education }\end{array}$ & Ofline & $\begin{array}{l}\text { Once per } \\
\text { month }\end{array}$ & Never, really & Paper books & $\$ 30-50$ Dollars & Gone with the Wind by Margaret Mitchell & $\begin{array}{l}\text { The Heroine's Journey: Woman's } \\
\text { Quest for Wholeness by Maureen } \\
\text { Murdock }\end{array}$ \\
\hline 43 & $\mathrm{~F}$ & 25 & $\begin{array}{l}\text { Full higher } \\
\text { education }\end{array}$ & Ofline & $\begin{array}{l}\text { Four times } \\
\text { per year }\end{array}$ & Yearly & Paper books & \$5-20 Dollars & Flowers for Algernon by Daniel Keyes & $\begin{array}{l}\text { Flowers for Algernon by Daniel } \\
\text { Keyes }\end{array}$ \\
\hline 44 & $\mathrm{~F}$ & 25 & $\begin{array}{l}\text { Full higher } \\
\text { education }\end{array}$ & Ofline & $\begin{array}{l}\text { Four times } \\
\text { per year }\end{array}$ & Yearly & Paper books & \$5-20 Dollars & $\begin{array}{l}\text { Conversations D'Un Enfant Du Siecle by } \\
\text { Frédéric Beigbeder }\end{array}$ & $\begin{array}{l}\text { Conversations D'Un Enfant Du } \\
\text { Siecle by Frédéric Beigbeder }\end{array}$ \\
\hline 45 & M & 25 & $\begin{array}{l}\text { Full higher } \\
\text { education }\end{array}$ & Ofline & $\begin{array}{l}\text { Once per } \\
\text { month }\end{array}$ & Never, really & Paper books & $\$ 30-50$ Dollars & Churchill: A Life by Martin Gilbert | & $\begin{array}{c}\text { The call sign «Cobra» by Erkebek } \\
\text { Abdulaev }\end{array}$ \\
\hline 46 & $\mathrm{~F}$ & 27 & $\begin{array}{l}\text { Full higher } \\
\text { education }\end{array}$ & $\begin{array}{l}\text { Online; } \\
\text { Ofline }\end{array}$ & $\begin{array}{l}\text { Once per } \\
\text { year }\end{array}$ & Never, really & E-books & \$5-20 Dollars & $\begin{array}{l}\text { The Ringing Cedars of Russia series by } \\
\text { Vladimir Megre }\end{array}$ & $\begin{array}{l}\text { History of Russian ballerina by } \\
\text { Anastasia Volochkova }\end{array}$ \\
\hline 47 & $\mathrm{~F}$ & 27 & $\begin{array}{l}\text { Full higher } \\
\text { education }\end{array}$ & Ofline & $\begin{array}{l}\text { Once per } \\
\text { month }\end{array}$ & Never, really & Paper books & $\$ 30-50$ Dollars & $\begin{array}{c}\text { Crime and Punishment by Fyodor } \\
\text { Dostoevsky }\end{array}$ & $\begin{array}{l}\text { A Decent Ride } \\
\text { by Irvine Welsh }\end{array}$ \\
\hline 48 & M & 27 & $\begin{array}{l}\text { Full higher } \\
\text { education }\end{array}$ & Ofline & $\begin{array}{l}\text { Once per } \\
\text { month }\end{array}$ & Never, really & Paper books & \$50-100 Dollars & Exodus by DJ Stalingrad & $\begin{array}{l}\text { Thinking, Fast and Slow by Daniel } \\
\text { Kahneman }\end{array}$ \\
\hline 49 & M & 27 & $\begin{array}{l}\text { Full higher } \\
\text { education }\end{array}$ & $\begin{array}{l}\text { Online; } \\
\text { Ofline }\end{array}$ & $\begin{array}{l}\text { four times } \\
\text { per year }\end{array}$ & Yearly & Paper books & $\$ 30-50$ Dollars & I am Maya Plisetskaya by Maya Plisetskaya & $\begin{array}{l}\text { Iris Apfel: Accidental Icon } \\
\text { by Iris Apfel }\end{array}$ \\
\hline 50 & $\mathrm{~F}$ & 28 & $\begin{array}{l}\text { Full higher } \\
\text { education }\end{array}$ & Ofline & $\begin{array}{l}\text { Once per } \\
\text { month }\end{array}$ & Never, really & Paper books & \$5-20 Dollars & $\begin{array}{l}\text { Nineteen Eighty-Four: A Novel by George } \\
\text { Orwell }\end{array}$ & Never Let Me Go by Kazuo Ishiguro \\
\hline 51 & $\mathrm{~F}$ & 28 & $\begin{array}{l}\text { Full higher } \\
\text { education }\end{array}$ & Ofline & $\begin{array}{l}\text { Four times } \\
\text { per year }\end{array}$ & Yearly & Paper books & \$50-100 Dollars & The Woman in the Dunes by Kōbō Abe & The Bells by Richard Harvell \\
\hline 52 & $\mathrm{~F}$ & 28 & $\begin{array}{l}\text { Full higher } \\
\text { education }\end{array}$ & $\begin{array}{l}\text { Online; } \\
\text { Ofline }\end{array}$ & $\begin{array}{l}\text { Four times } \\
\text { per year }\end{array}$ & Yearly & Paper books & \$50-100 Dollars & The Bible & Rosehip necklace by Irina Savka \\
\hline 53 & M & 28 & $\begin{array}{l}\text { Full higher } \\
\text { education }\end{array}$ & Ofline & $\begin{array}{l}\text { Four times } \\
\text { per year }\end{array}$ & Never, really & Paper books & \$5-20 Dollars & Man's Search for Meaning by Viktor Frankl & The City by Valerian Pidmohylny \\
\hline 54 & $\mathrm{~F}$ & 29 & $\begin{array}{l}\text { Full higher } \\
\text { education }\end{array}$ & Ofline & $\begin{array}{l}\text { Four times } \\
\text { per year }\end{array}$ & Never, really & E-books & \$5-20 Dollars & $\begin{array}{l}\text { All Quiet on the Western Front by Erich } \\
\text { Maria Remarque }\end{array}$ & $\begin{array}{c}\text { A Clockwork Orange by Anthony } \\
\text { Burgess }\end{array}$ \\
\hline 55 & M & 29 & $\begin{array}{l}\text { Full higher } \\
\text { education }\end{array}$ & Ofline & $\begin{array}{l}\text { Four times } \\
\text { per year }\end{array}$ & Never, really & $\begin{array}{l}\text { Paper books, } \\
\text { e-books }\end{array}$ & \$5-20 Dollars & $\begin{array}{l}\text { Escape from Camp 14: One man's } \\
\text { remarkable odyssey from North Korea to } \\
\text { freedom in the West Reprints by Blaine } \\
\text { Harden }\end{array}$ & $\begin{array}{l}\text { Nicholas Fandorin series (about } \\
\text { Erast Fandorin's grandson, a } \\
\text { modern-day British historian) by } \\
\text { Boris Akunin }\end{array}$ \\
\hline 56 & M & 29 & $\begin{array}{l}\text { Full higher } \\
\text { education }\end{array}$ & Ofline & $\begin{array}{l}\text { Four times } \\
\text { per year }\end{array}$ & Never, really & Paper books & \$30-50 Dollars & The Lord of the Rings by J. R. R. Tolkien & La Magdalena by William M. Valtos \\
\hline 57 & $\mathrm{~F}$ & 30 & $\begin{array}{l}\text { Full higher } \\
\text { education }\end{array}$ & Ofline & $\begin{array}{l}\text { Once per } \\
\text { month }\end{array}$ & Never, really & Paper books & $\$ 30-50$ Dollars & The Catcher in the Rye by J. D. Salinger & Notebooks by Sergei Dovlatov \\
\hline 58 & $\mathrm{~F}$ & 30 & $\begin{array}{l}\text { Full higher } \\
\text { education }\end{array}$ & Online & $\begin{array}{l}\text { Four times } \\
\text { per year }\end{array}$ & Never, really & E-books & $\$ 30-50$ Dollars & $\begin{array}{l}\text { Cassandra's Path, or Adventures with } \\
\text { Macaroni by Julia Voznesenskaya }\end{array}$ & $\begin{array}{l}\text { The Rape of Europe by Evgeny } \\
\text { Vodolazkin }\end{array}$ \\
\hline 59 & $\mathrm{~F}$ & 31 & $\begin{array}{l}\text { Full higher } \\
\text { education }\end{array}$ & Ofline & $\begin{array}{l}\text { Once per } \\
\text { year }\end{array}$ & Never, really & Paper books & $\$ 30-50$ Dollars & $\begin{array}{c}\text { Apply Your Problem Solving Know How by } \\
\text { Dale Carnegie Forgive myself by Luule } \\
\text { Viilma }\end{array}$ & The Art of Being by Erich Fromm \\
\hline 60 & $\mathrm{~F}$ & 31 & $\begin{array}{l}\text { Full higher } \\
\text { education }\end{array}$ & Ofline & $\begin{array}{l}\text { Four times } \\
\text { per year }\end{array}$ & Never, really & Paper books & $\$ 30-50$ Dollars & $\begin{array}{c}\text { Crime and Punishment by Fyodor } \\
\text { Dostoevsky }\end{array}$ & $\begin{array}{l}\text { Totem and Taboo by Sigmund } \\
\text { Freud }\end{array}$ \\
\hline 61 & $\mathrm{~F}$ & 31 & $\begin{array}{l}\text { Full higher } \\
\text { education }\end{array}$ & Ofline & $\begin{array}{l}\text { Four times } \\
\text { per year }\end{array}$ & Never, really & Paper books & \$50-100 Dollars & $\begin{array}{c}\text { My Grandmother Asked Me to Tell You } \\
\text { She's Sorry } \\
\text { by Fredrik Backman }\end{array}$ & $\begin{array}{l}\text { Britt-Marie Was Here by Fredrik } \\
\text { Backman }\end{array}$ \\
\hline 62 & F & 31 & $\begin{array}{l}\text { Full higher } \\
\text { education }\end{array}$ & $\begin{array}{l}\text { Online; } \\
\text { Ofline }\end{array}$ & $\begin{array}{l}\text { Once per } \\
\text { month }\end{array}$ & Never, really & $\begin{array}{l}\text { Paper books, } \\
\text { e-books }\end{array}$ & \$5-20 Dollars & The Rosy Crucifixion by Henry Miller & $\begin{array}{c}\text { The Inner World Outside Object } \\
\text { Relations Theory and Psychodrama } \\
\text { by Paul Holmes }\end{array}$ \\
\hline
\end{tabular}




\begin{tabular}{|c|c|c|c|c|c|c|c|c|c|c|}
\hline ID & $\begin{array}{l}\text { Gender } \\
\text { Female/ } \\
\text { Male }\end{array}$ & Age & Education & $\begin{array}{l}\text { Do you purchase } \\
\text { books at online or } \\
\text { ofline bookstores }\end{array}$ & $\begin{array}{l}\text { How often } \\
\text { Do you } \\
\text { purchase } \\
\text { books }\end{array}$ & $\begin{array}{l}\text { How often do } \\
\text { you visit your } \\
\text { local library }\end{array}$ & $\begin{array}{l}\text { Do you prefer } \\
\text { paper books or } \\
\text { E-books }\end{array}$ & $\begin{array}{l}\text { How much money } \\
\text { are you ready to } \\
\text { spend on book per } \\
\text { one time }\end{array}$ & $\begin{array}{l}\text { Life-changing book } \\
\text { That shaped your thinking }\end{array}$ & $\begin{array}{l}\text { Book, you've } \\
\text { Recently read }\end{array}$ \\
\hline 63 & $\mathrm{~F}$ & 32 & $\begin{array}{l}\text { Full higher } \\
\text { education }\end{array}$ & Online & $\begin{array}{l}\text { Once per } \\
\text { month }\end{array}$ & Never, really & Paper books & $\$ 30-50$ Dollars & $\begin{array}{c}\text { The Dolphin Way: A Parent's Guide to } \\
\text { Raising Healthy, Happy, and Motivated } \\
\text { Kids-Without Turning into a Tiger by Shimi } \\
\text { K. Kang }\end{array}$ & Final Exam by Julio Cortázar \\
\hline 64 & $\mathrm{~F}$ & 32 & $\begin{array}{l}\text { Full higher } \\
\text { education }\end{array}$ & Online & $\begin{array}{l}\text { Once per } \\
\text { month }\end{array}$ & Monthly & Paper books & $\$ 30-50$ Dollars & $\begin{array}{c}\text { Confessions of an Advertising Man by David } \\
\text { Mackenzie Ogilvy }\end{array}$ & Steppenwolf by Hermann Hesse \\
\hline 65 & $\mathrm{~F}$ & 32 & $\begin{array}{l}\text { Full higher } \\
\text { education }\end{array}$ & Ofline & $\begin{array}{l}\text { Once per } \\
\text { year }\end{array}$ & Never, really & Paper books & \$5-20 Dollars & $\begin{array}{c}\text { The Master and Margarita by Mikhail } \\
\text { Bulgakov }\end{array}$ & Misèrere by Jean-Christophe Grangé \\
\hline 66 & $\mathrm{~F}$ & 33 & $\begin{array}{l}\text { Full higher } \\
\text { education }\end{array}$ & Ofline & $\begin{array}{l}\text { Once per } \\
\text { month }\end{array}$ & Never, really & Paper books & \$50-100 Dollars & Atlas Shrugged by Ayn Rand & $\begin{array}{c}\text { The Power of Now: A Guide to } \\
\text { Spiritual Enlightenment by Eckhart } \\
\text { Tolle }\end{array}$ \\
\hline 67 & $\mathrm{~F}$ & 33 & $\begin{array}{l}\text { Full higher } \\
\text { education }\end{array}$ & Ofline & $\begin{array}{l}\text { Four times } \\
\text { per year }\end{array}$ & Never, really & Paper books & $\$ 30-50$ Dollars & Garden of Gethsemane by Ivan Bahrianyi & Whiffet by Vitaly Zapeka \\
\hline 68 & M & 33 & $\begin{array}{l}\text { Full higher } \\
\text { education }\end{array}$ & Online & $\begin{array}{l}\text { Once per } \\
\text { year }\end{array}$ & Never, really & E-books & $\$ 30-50$ Dollars & $\begin{array}{l}\text { Hands-On Reactive Programming in Spring } \\
5 \text { by Oleh Dokuka, Igor Lozynskyi }\end{array}$ & ${ }^{2}$ \\
\hline 69 & M & 33 & $\begin{array}{l}\text { Full higher } \\
\text { education }\end{array}$ & Ofline & $\begin{array}{l}\text { Four times } \\
\text { per year }\end{array}$ & Yearly & E-books & \$50-100 Dollars & $\begin{array}{c}\text { Thus Spoke Zarathustra by Friedrich } \\
\text { Nietzsche }\end{array}$ & $\begin{array}{l}\text { Poems about Europe by Antonin } \\
\text { Ladinsky }\end{array}$ \\
\hline 70 & M & 33 & $\begin{array}{l}\text { Full higher } \\
\text { education }\end{array}$ & Ofline & $\begin{array}{l}\text { Four times } \\
\text { per year }\end{array}$ & Never, really & Paper books & \$50-100 Dollars & $\begin{array}{l}\text { A Brief History of the Universe by J.P. } \\
\text { McEvoy }\end{array}$ & $\begin{array}{l}\text { Norse Mythology } \\
\text { by Neil Gaiman }\end{array}$ \\
\hline 71 & M & 33 & $\begin{array}{l}\text { The PhD in } \\
\text { History }\end{array}$ & Ofline & $\begin{array}{l}\text { Four times } \\
\text { per year }\end{array}$ & Weekly & Paper books & \$5-20 Dollars & The Diary of a Bookseller by Shaun Bythell & $\begin{array}{l}\text { Conversation about the ancient } \\
\text { world by Gregory Skovoroda }\end{array}$ \\
\hline 72 & $\mathrm{~F}$ & 35 & $\begin{array}{l}\text { Full higher } \\
\text { education }\end{array}$ & Online & $\begin{array}{l}\text { Once per } \\
\text { week }\end{array}$ & Never, really & $\begin{array}{l}\text { Paper books, } \\
\text { e-books }\end{array}$ & \$50-100 Dollars & $\begin{array}{l}\text { Breaking the Habit of Being Yourself by Dr } \\
\text { Joe Dispenza }\end{array}$ & $\begin{array}{l}\text { Das große Buch von Koko und Kiri } \\
\text { by Erwin Moser }\end{array}$ \\
\hline 73 & M & 35 & $\begin{array}{l}\text { Full higher } \\
\text { education }\end{array}$ & Online & $\begin{array}{l}\text { Four times } \\
\text { per year }\end{array}$ & Never, really & E-books & \$50-100 Dollars & The Book of Mirdad by Mikha'il Na'ima & $\begin{array}{l}\text { The Casual Vacancy by J.K. } \\
\text { Rowling }\end{array}$ \\
\hline 74 & M & 35 & $\begin{array}{l}\text { Full higher } \\
\text { education }\end{array}$ & Ofline & $\begin{array}{l}\text { Once per } \\
\text { year }\end{array}$ & Never, really & Paper books & \$5-20 Dollars & $\begin{array}{l}\text { Reality Transurfing 1: The Space of } \\
\text { Variations by Vadim Zeland }\end{array}$ & $\begin{array}{c}\text { Eugénie Grandet by Honoré de } \\
\text { Balzac }\end{array}$ \\
\hline 75 & $\mathrm{~F}$ & 36 & $\begin{array}{l}\text { Full higher } \\
\text { education }\end{array}$ & Ofline & $\begin{array}{l}\text { Once per } \\
\text { month }\end{array}$ & Never, really & Paper books & \$5-20 Dollars & $\begin{array}{c}\text { In Search of the City of the Gods Volume III } \\
\text { - In the Arms of Shambhala by Ernst } \\
\text { Muldašev }\end{array}$ & $\begin{array}{l}\text { The King of Warsaw: A Novel by } \\
\text { Szczepan Twardoch }\end{array}$ \\
\hline 76 & $\mathrm{~F}$ & 39 & $\begin{array}{l}\text { Full higher } \\
\text { education }\end{array}$ & Ofline & $\begin{array}{l}\text { Once per } \\
\text { year }\end{array}$ & Yearly & Paper books & $\$ 30-50$ Dollars & $\begin{array}{l}\text { Words that Ring Through Time: The Fifty } \\
\text { Most Important Speeches in History and } \\
\text { How they Changed Our World by Terry } \\
\text { Golway }\end{array}$ & $\begin{array}{c}\text { The Fifth Symphony by Jevhen } \\
\text { Malanjuk }\end{array}$ \\
\hline 77 & $\mathrm{~F}$ & 40 & $\begin{array}{l}\text { Full higher } \\
\text { education }\end{array}$ & Ofline & $\begin{array}{l}\text { Four times } \\
\text { per year }\end{array}$ & Never, really & $\begin{array}{l}\text { Paper books, } \\
\text { e-books }\end{array}$ & \$50-100 Dollars & Nineteen Eighty-Four by George Orwell & Animal Farm by George Orwell \\
\hline 78 & F & 40 & $\begin{array}{l}\text { Full higher } \\
\text { education }\end{array}$ & Ofline & $\begin{array}{l}\text { Four times } \\
\text { per year }\end{array}$ & Never, really & $\begin{array}{l}\text { Paper books, } \\
\text { e-books }\end{array}$ & $\$ 30-50$ Dollars & The Thirteenth Tale by Diane Setterfield & $\begin{array}{l}\text { Bellman \& Black by Diane } \\
\text { Setterfield }\end{array}$ \\
\hline 79 & M & 40 & $\begin{array}{l}\text { Full higher } \\
\text { education }\end{array}$ & Online & $\begin{array}{l}\text { Four times } \\
\text { per year }\end{array}$ & Never, really & Paper books & \$50-100 Dollars & Marketing Warfare by Al Ries, Jack Trout & $\begin{array}{l}\text { Red Pill. Look the truth in the eyes } \\
\text { by Andrei Kurpatov }\end{array}$ \\
\hline 80 & M & 41 & $\begin{array}{l}\text { Full higher } \\
\text { education }\end{array}$ & Online & $\begin{array}{l}\text { Once per } \\
\text { year }\end{array}$ & Never, really & E-books & \$5-20 Dollars & Light in August by William Faulkner & $\begin{array}{l}\text { The Howling Miller by Arto Tapio } \\
\text { Paasilinna }\end{array}$ \\
\hline 81 & M & 41 & $\begin{array}{l}\text { Full higher } \\
\text { education }\end{array}$ & Ofline & $\begin{array}{l}\text { Once per } \\
\text { month }\end{array}$ & Never, really & Paper books & $\$ 30-50$ Dollars & Eneyida by Ivan Kotlyarevsky & Kobzar by Taras Shevchenko \\
\hline 82 & F & 44 & $\begin{array}{l}\text { Full higher } \\
\text { education }\end{array}$ & Ofline & $\begin{array}{l}\text { No } \\
\text { determinable } \\
\text { frequency }\end{array}$ & Never, really & $\begin{array}{l}\text { Paper books, } \\
\text { e-books }\end{array}$ & \$50-100 Dollars & $\begin{array}{c}\text { The Adventures of Huckleberry Finn by } \\
\text { Mark Twain }\end{array}$ & $\begin{array}{l}\text { Ragnarok: The End of the Gods by } \\
\text { Antonia Susan Byatt }\end{array}$ \\
\hline 83 & $\mathrm{~F}$ & 44 & $\begin{array}{l}\text { Full higher } \\
\text { education }\end{array}$ & Ofline & $\begin{array}{l}\text { Four times } \\
\text { per year }\end{array}$ & Never, really & Paper books & $\$ 30-50$ Dollars & $\begin{array}{c}\text { Men Are from Mars, Women Are from } \\
\text { Venus by John Gray }\end{array}$ & $\begin{array}{l}\text { What You Feel You Can Heal by } \\
\text { John Gray }\end{array}$ \\
\hline
\end{tabular}




\begin{tabular}{|c|c|c|c|c|c|c|c|c|c|c|}
\hline ID & $\begin{array}{l}\text { Gender } \\
\text { Female/ } \\
\text { Male }\end{array}$ & Age & Education & $\begin{array}{l}\text { Do you purchase } \\
\text { books at online or } \\
\text { ofline bookstores }\end{array}$ & $\begin{array}{l}\text { How often } \\
\text { Do you } \\
\text { purchase } \\
\text { books }\end{array}$ & $\begin{array}{l}\text { How often do } \\
\text { you visit your } \\
\text { local library }\end{array}$ & $\begin{array}{l}\text { Do you prefer } \\
\text { paper books or } \\
\text { E-books }\end{array}$ & $\begin{array}{l}\text { How much money } \\
\text { are you ready to } \\
\text { spend on book per } \\
\text { one time }\end{array}$ & $\begin{array}{l}\text { Life-changing book } \\
\text { That shaped your thinking }\end{array}$ & $\begin{array}{l}\text { Book, you've } \\
\text { Recently read }\end{array}$ \\
\hline 84 & $\mathrm{~F}$ & 44 & $\begin{array}{l}\text { Full higher } \\
\text { education }\end{array}$ & Online & $\begin{array}{l}\text { Four times } \\
\text { per year }\end{array}$ & Never, really & $\begin{array}{l}\text { Paper books, } \\
\text { e-books }\end{array}$ & $\$ 30-50$ Dollars & $\begin{array}{l}\text { One Hundred Years of Solitude by Gabriel } \\
\text { García Márquez }\end{array}$ & $\begin{array}{l}\text { Manipulation of the word in the } \\
\text { media by Anna Danilova }\end{array}$ \\
\hline 85 & $\mathrm{~F}$ & 45 & $\begin{array}{l}\text { Full higher } \\
\text { education }\end{array}$ & Ofline & $\begin{array}{l}\text { Once per } \\
\text { year }\end{array}$ & Never, really & Paper books & $\$ 30-50$ Dollars & $\begin{array}{c}\text { Kiss That Frog!: } 21 \text { Great Ways to Turn } \\
\text { Negatives into Positives in Your Life and } \\
\text { Work by Brian Tracy }\end{array}$ & $\begin{array}{l}\text { No Excuses!: The Power of Self- } \\
\text { Discipline by Brian Tracy }\end{array}$ \\
\hline 86 & $\mathrm{~F}$ & 46 & $\begin{array}{l}\text { Full higher } \\
\text { education }\end{array}$ & Ofline & $\begin{array}{l}\text { Once per } \\
\text { year }\end{array}$ & Never, really & $\begin{array}{l}\text { Paper books, e- } \\
\text { books }\end{array}$ & \$50-100 Dollars & The Summer Book by Tove Jansson & $\begin{array}{l}\text { Black: The History of a Color by } \\
\text { Michel Pastoureau }\end{array}$ \\
\hline 87 & $\mathrm{~F}$ & 49 & $\begin{array}{l}\text { Full higher } \\
\text { education }\end{array}$ & Ofline & $\begin{array}{l}\text { Once per } \\
\text { year }\end{array}$ & Never, really & Paper books & \$50-100 Dollars & $\begin{array}{l}\text { No B.S. Ruthless Management of People and } \\
\text { Profits by Dan Kennedy }\end{array}$ & $\begin{array}{l}\text { The Curious Case of Benjamin } \\
\text { Button by Francis Fitzgerald }\end{array}$ \\
\hline 88 & M & 53 & $\begin{array}{l}\text { Full higher } \\
\text { education }\end{array}$ & Ofline & $\begin{array}{l}\text { Once per } \\
\text { week }\end{array}$ & Never, really & Paper books & $\$ 30-50$ Dollars & Jo confesso by Jaume Cabré & Il giorno by Giuseppe Parini \\
\hline 89 & M & 54 & $\begin{array}{l}\text { Full higher } \\
\text { education }\end{array}$ & Ofline & $\begin{array}{l}\text { Once per } \\
\text { month }\end{array}$ & Yearly & Paper books & $\$ 30-50$ Dollars & $\begin{array}{l}\text { Think and Grow Rich } \\
\text { by Napoleon Hill }\end{array}$ & $\begin{array}{l}\text { Plato, Not Prozac!: Applying } \\
\text { Eternal Wisdom to Everyday } \\
\text { Problems by Lou Marinoff PhD }\end{array}$ \\
\hline 90 & M & 54 & $\begin{array}{l}\text { Full higher } \\
\text { education }\end{array}$ & Ofline & $\begin{array}{l}\text { Four times } \\
\text { per year }\end{array}$ & Monthly & Paper books & \$5-20 Dollars & $\begin{array}{c}\text { Everything is in your hands by Oleksandr } \\
\text { Shovkovskyi }\end{array}$ & $\begin{array}{c}\text { Pep's City: The Making of a } \\
\text { Superteam by by Lu Martin , Pol } \\
\text { Ballus }\end{array}$ \\
\hline 91 & M & 57 & $\begin{array}{l}\text { Full higher } \\
\text { education }\end{array}$ & Ofline & $\begin{array}{l}\text { Once per } \\
\text { week }\end{array}$ & Never, really & Paper books & \$50-100 Dollars & $\begin{array}{l}\text { The Complete Essays of Michel de } \\
\text { Montaigne by Michel de Montaigne }\end{array}$ & $\begin{array}{l}\text { Strategy: The Logic of War and } \\
\text { Peace by Edward N. Luttwak }\end{array}$ \\
\hline 92 & $\mathrm{~F}$ & 57 & $\begin{array}{l}\text { Full higher } \\
\text { education }\end{array}$ & Ofline & $\begin{array}{l}\text { Once per } \\
\text { month }\end{array}$ & Yearly & Paper books & \$5-20 Dollars & Still Me by Jojo Moyes & $\begin{array}{l}\text { The Giver of Stars } \\
\text { by Jojo Moyes }\end{array}$ \\
\hline 93 & $\mathrm{~F}$ & 58 & $\begin{array}{l}\text { Full higher } \\
\text { education }\end{array}$ & Ofline & $\begin{array}{l}\text { Once per } \\
\text { month }\end{array}$ & Monthly & $\begin{array}{l}\text { Paper books, } \\
\text { e-books }\end{array}$ & \$30-50 Dollars & $\begin{array}{c}\text { The Woman Who Borrowed Memories by } \\
\text { Tove Jansson }\end{array}$ & $\begin{array}{l}\text { Tove Jansson: Work and Love } \\
\text { by Tuula Karjalainen }\end{array}$ \\
\hline 94 & M & 59 & $\begin{array}{l}\text { Full higher } \\
\text { education }\end{array}$ & Ofline & $\begin{array}{l}\text { Once per } \\
\text { month }\end{array}$ & Never, really & Paper books & \$50-100 Dollars & $\begin{array}{l}\text { BaZi- The Destiny Code: Your Guide to the } \\
\text { Four Pillars of Destiny by Joey Yap }\end{array}$ & $\begin{array}{c}\text { Four Pillars of Destiny Exploring } \\
\text { Water Charts } \\
\text { by Dr Jin Peh }\end{array}$ \\
\hline 95 & M & 61 & $\begin{array}{l}\text { Full higher } \\
\text { education }\end{array}$ & Ofline & $\begin{array}{l}\text { Four times } \\
\text { per year }\end{array}$ & Never, really & Paper books & $\$ 30-50$ Dollars & The Bible & Vladimir Shcherbakov \\
\hline 96 & $\mathrm{~F}$ & 64 & $\begin{array}{l}\text { Full higher } \\
\text { education }\end{array}$ & Ofline & $\begin{array}{l}\text { Four times } \\
\text { per year }\end{array}$ & Monthly & Paper books & $\$ 30-50$ Dollars & $\begin{array}{l}\text { Thoughts on Art and Life } \\
\text { by Leonardo da Vinci }\end{array}$ & $\begin{array}{l}\text { Suite and partita in the piano art of } \\
\text { Ukrainian composers of the } \\
\text { twentieth century by Maria } \\
\text { Kalashnik }\end{array}$ \\
\hline 97 & M & 64 & $\begin{array}{l}\text { Full higher } \\
\text { education }\end{array}$ & Ofline & $\begin{array}{l}\text { Once per } \\
\text { month }\end{array}$ & Monthly & Paper books & $\$ 30-50$ Dollars & $\begin{array}{l}\text { A Year in Provence } \\
\text { by Peter Mayle }\end{array}$ & $\begin{array}{c}\text { Encore Provence: New Adventures } \\
\text { in the South of France by Peter } \\
\text { Mayle }\end{array}$ \\
\hline 98 & $\mathrm{~F}$ & 65 & $\begin{array}{l}\text { Full higher } \\
\text { education }\end{array}$ & Online & $\begin{array}{l}\text { Once per } \\
\text { month }\end{array}$ & Never, really & $\begin{array}{l}\text { Paper books,e- } \\
\text { books }\end{array}$ & \$50-100 Dollars & Three Comrades by Erich Maria Remarque & $\begin{array}{c}\text { Oedipus of Moscow by Valery } \\
\text { Primost }\end{array}$ \\
\hline 99 & $\mathrm{~F}$ & 70 & $\begin{array}{l}\text { Full higher } \\
\text { education }\end{array}$ & Ofline & $\begin{array}{l}\text { Once per } \\
\text { month }\end{array}$ & Never, really & Paper books & \$5-20 Dollars & $\begin{array}{c}\text { Some Buried Caesar/The Golden Spiders } \\
\text { (Nero Wolfe) } \\
\text { by Rex Stout }\end{array}$ & $\begin{array}{l}\text { Nine Coaches Waiting } \\
\text { by Mary Stewart }\end{array}$ \\
\hline 100 & $\mathrm{~F}$ & 71 & $\begin{array}{l}\text { Full higher } \\
\text { education }\end{array}$ & Ofline & $\begin{array}{l}\text { Four times } \\
\text { per year }\end{array}$ & Never, really & Paper books & $\$ 30-50$ Dollars & $\begin{array}{l}\text { The God Equation: The Quest for a Theory } \\
\text { of Everything } \\
\text { by Michio Kaku }\end{array}$ & $\begin{array}{l}\text { A Year in Provence } \\
\text { by Peter Mayle }\end{array}$ \\
\hline
\end{tabular}




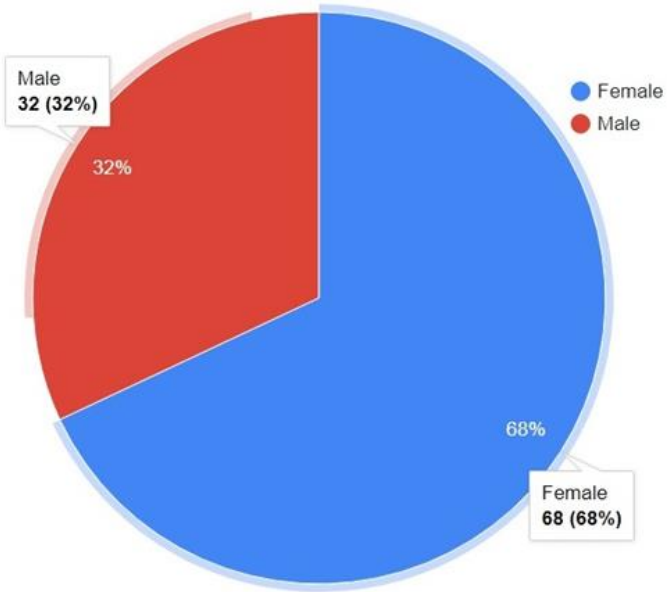

Fig. 1. Result of the questionnaire. Demographic Information about respondents. Gender. Source: author's elaboration.

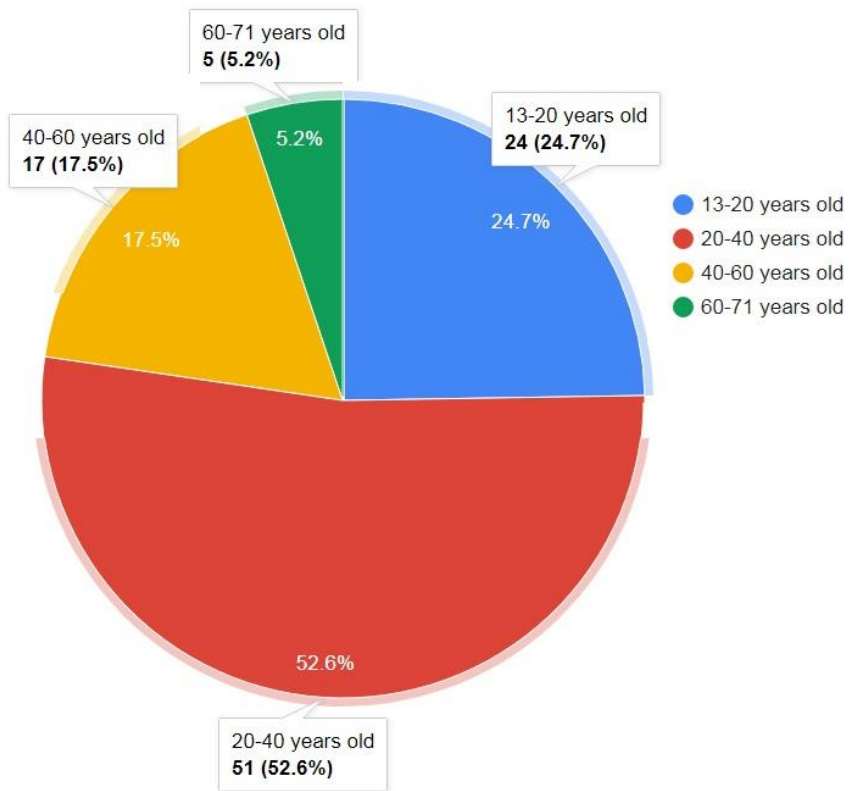

Fig. 2. Result of the questionnaire. Demographic Information about respondents. Age. Source: author's elaboration.

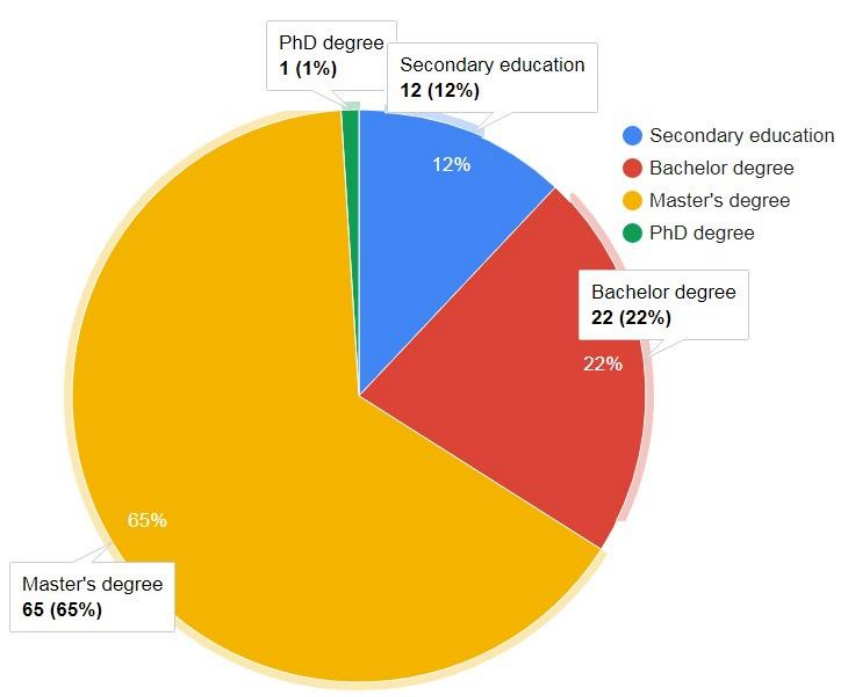

Fig. 3. Result of the questionnaire. Demographic Information about respondents. Education level. Source: author's elaboration.

Results show us that the largest pieces of the pie come from readers whose book-buying budged amounts to $30-50 \$$ per once purchase (42\%), 33\% respondents ready to pay once for the book $50-20 \$$, and only $25 \%$ are the richest - they can pay
50-100\$ per book. So, respondents with a high financial level more often bought books than respondents with a low financial level.

The results on Fig. 5 show us that the overwhelming majority of readers $(75 \%)$ prefer to purchase books offline, in a bookstore. Online book shopping is the prerogative of $15 \%$ respondents. Both - online \& offline bookstores chose $10 \%$ of the survey respondents.

By answering on the following question "how often they purchase books", $35 \%$ respondents pointed to four times per year, $34 \%$ respondents purchase books once per month, $22 \%$ once per year, $6 \%$ once per week and $3 \%$ respondents chose "no determinable" (Fig. 6.).

Residents buy printed books (74\%) slightly more often than e-books (12\%). Both - printed and e-book buy only $14 \%$ residents (Fig. 7.).

Answering the question how often they borrow books from a library, $14,1 \%$ of respondents answered that they visit library every month, 17,2\% every year and 68,7\% never used library services and resources (Fig. 8.).

13-20 years old 40-60 years old people more often than those from other groups bought borrowed books from a library. School and college students more often than others downloaded books for free, and also borrowed them from a school library.

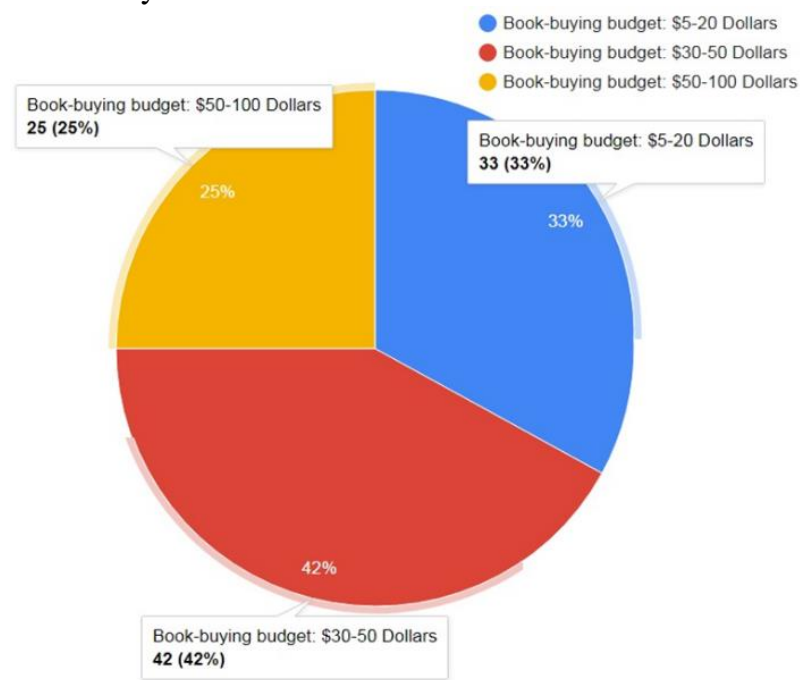

Fig. 4. Result of the questionnaire. Book-buying budget. Source: author's elaboration.

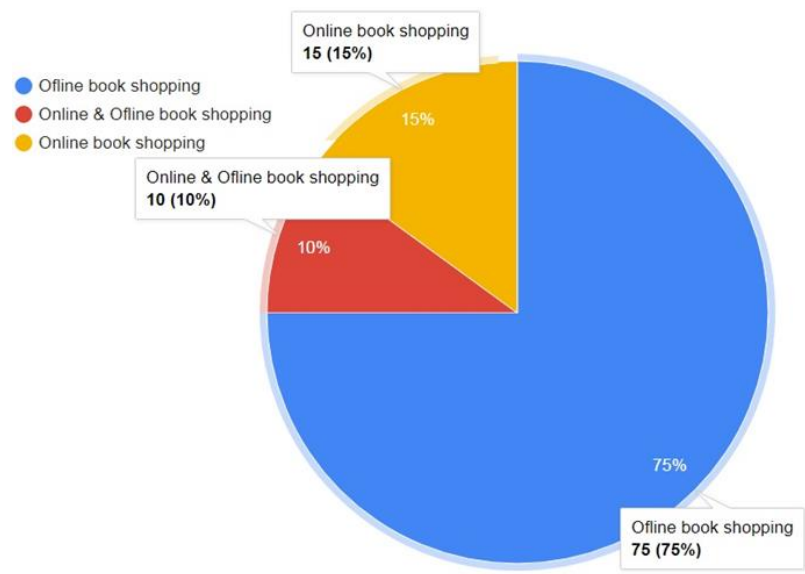

Fig. 5. Result of the questionnaire. Statistical data about publishing preferences of respondents. Book shopping. Source: author's elaboration. 


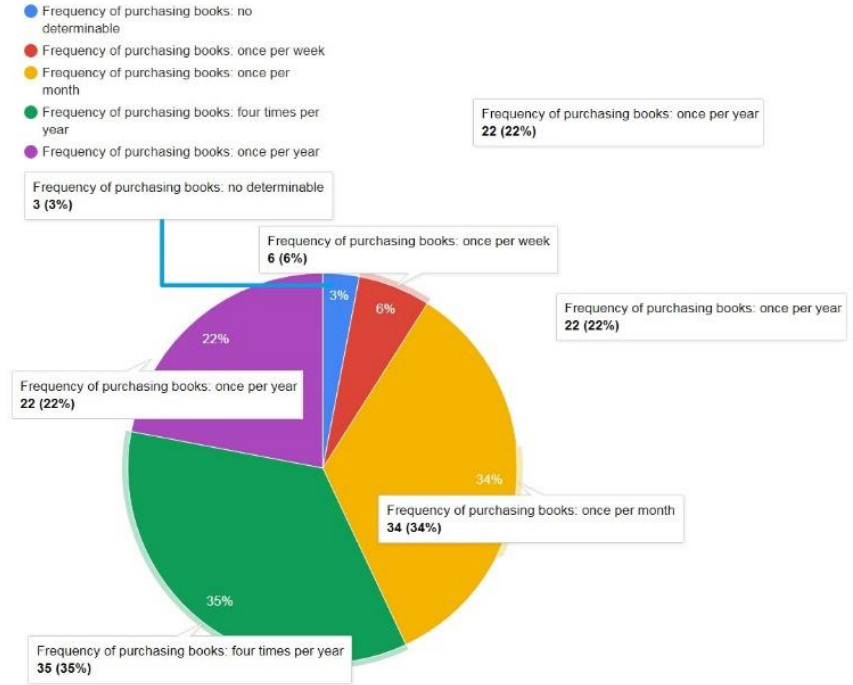

Fig. 6. Result of the questionnaire. Statistical data about publishing preferences of respondents. Frequency of book purchasing. Source: author's elaboration.

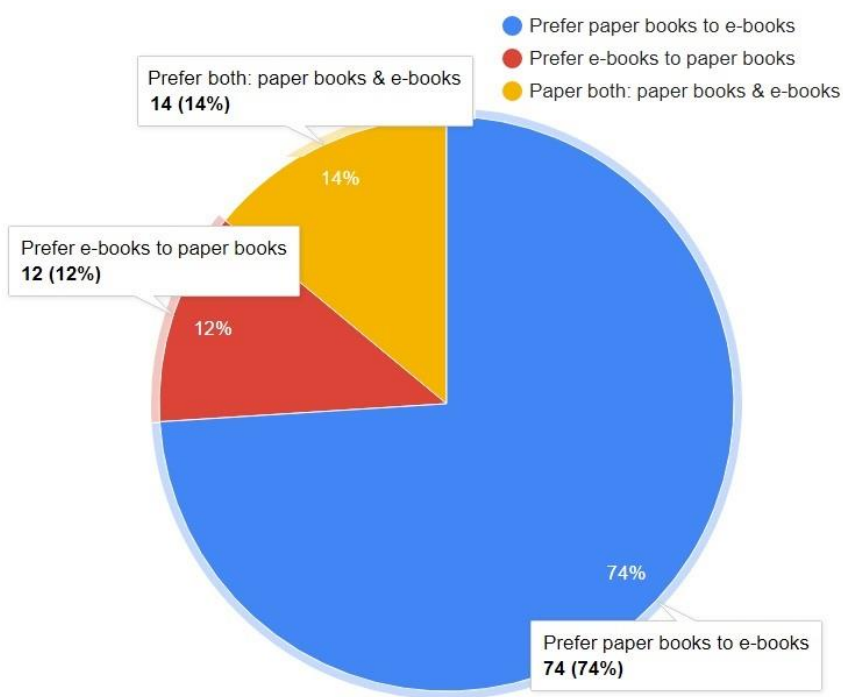

Fig. 7. Result of the questionnaire. Statistical data about publishing preferences of respondents. Paper book vs e-book. Source: author's elaboration.

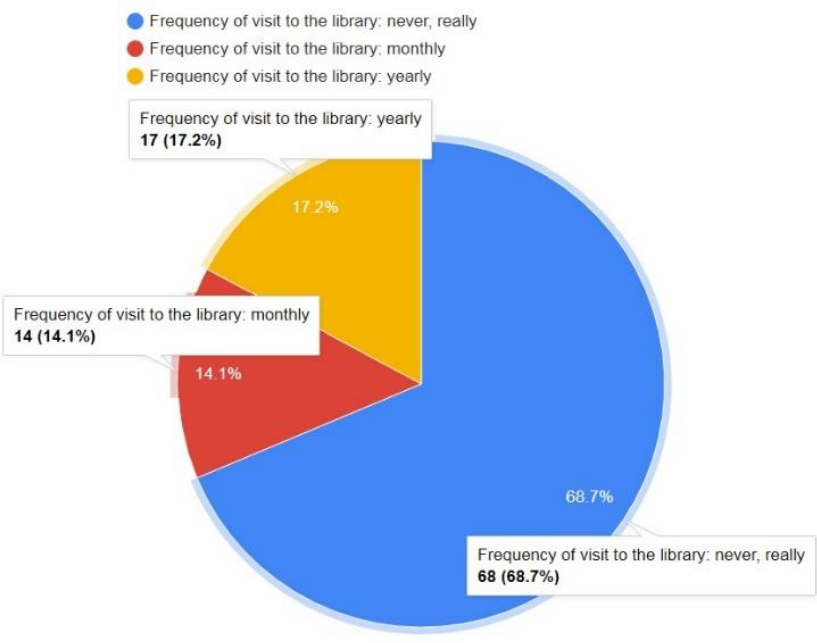

Fig. 8. Result of the questionnaire. Statistical data about publishing preferences of respondents. Library visiting. Source: author's elaboration.
As we can see on Fig. 9, the most popular among Ukrainian readers are such nonfiction genres: Self-Help/Personal Development (15,2\%), Motivational/Inspirational (14,3\%). During the last year $8 \%$ of respondents read Health \& Fitness, Education \& Teaching books. The most unpopular genres among respondents are Religion \& Spirituality $(1,5 \%)$ and Law \& Criminology $(0,9 \%)$. It should be noted that the level of popularity of various genres differs significantly among different groups of respondents. In particular, the popularity of different genres among young and old respondents is quite different. The picture of fiction genre popularity looks this way (Fig. 10.): Adventure (20,1\%), Fantasy (15\%), Historical Fiction and Romance (13,9\%), Detective \& Mystery (13,3\%), Dystopian $(8,6 \%)$, Science Fiction $(5,9 \%)$, Horror $(5,6 \%)$, Children's Fiction (5,4\%), Thriller $(4,2 \%)$, Young Adult $(3,7 \%)$.

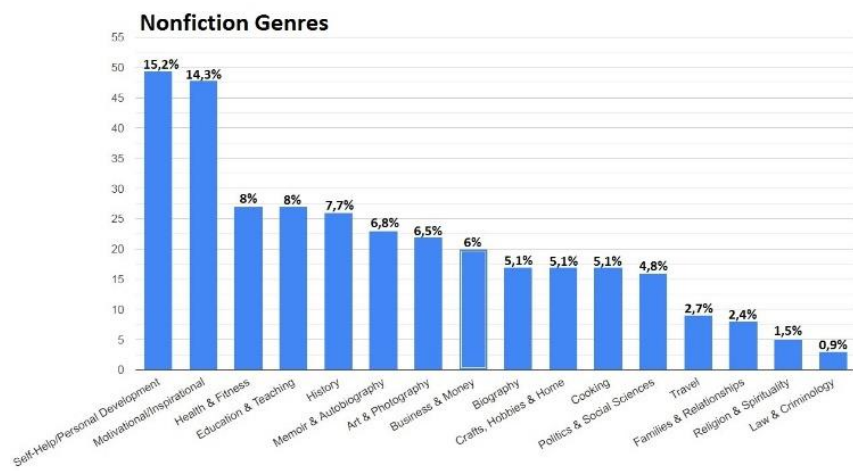

Fig. 9. Result of the questionnaire. Statistical data about preferences of nonfiction genres. Source: author's elaboration.

\section{Fiction Genres}

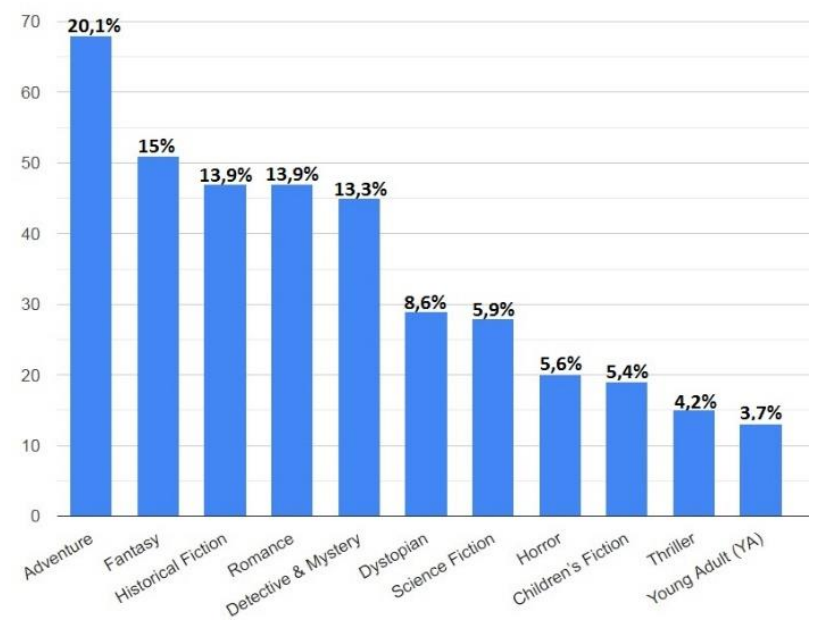

Fig. 10. Result of the questionnaire. Statistical data about preferences of fiction genres. Source: author's elaboration.

When answering the open-ended questions "Which lifechanging books that shaped your thinking?" and "Which book you've recently read?" 6 times respondents named John Green, 4 times respondents named George Orwell, 3 times respondents named Dale Carnegie, Daniel Keyes, Erich Maria Remarque, J. D. Salinger, Jojo Moyes, Peter Mayle; 2 times respondents named Albert Camus, Antoine de SaintExupéry , Arthur Conan Doyle, Ayn Rand, Bernard Werber, Brian Tracy, Daniel Goleman, Diane Setterfield, Erin Hunter, Frédéric Beigbeder, Fredrik Backman, Fyodor Dostoevsky, Gregory David Roberts, Joe Dispenza, John Gray, Margaret Mitchell, Neil Gaiman, Peter Drucker, Richard Templar, Tove Jansson. So, John Green was among the top (Fig. 11). 


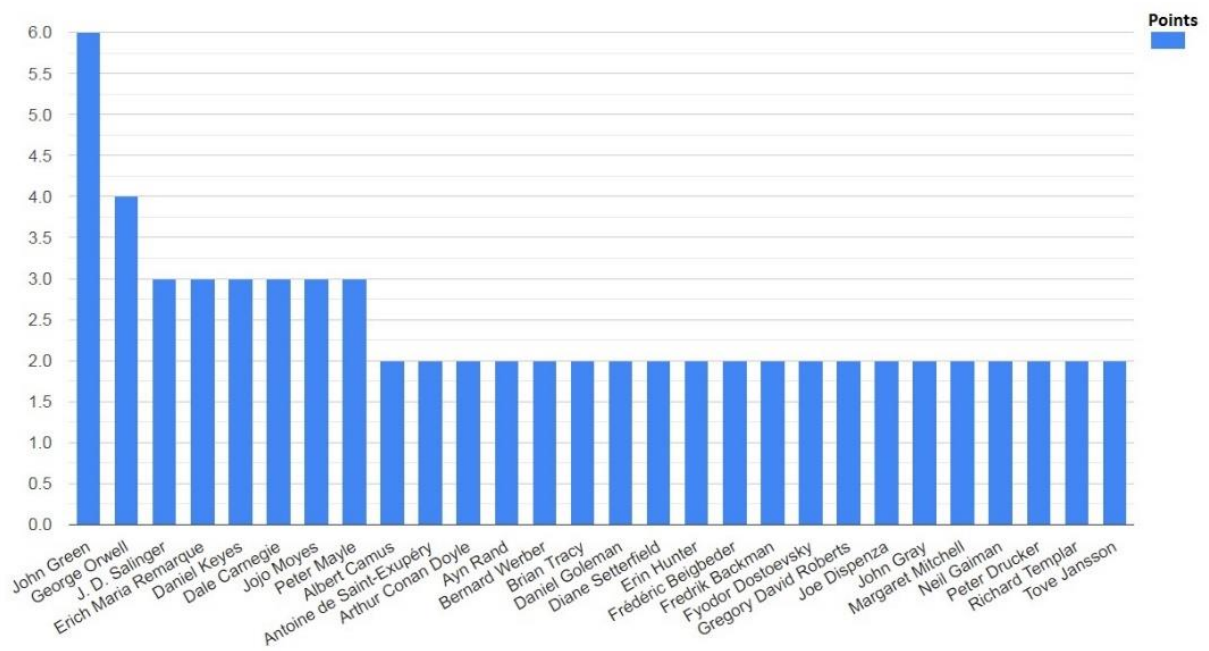

Fig. 11. Result of the questionnaire. Statistical data about most popular writers among respondents. Source: author's elaboration.

\section{CONCLUSION}

Based on the data and discussion above, the conclusions are as follows. Books are easy to access without leaving home. It's important fact considering coronavirus outbreak. The results of this study show, first, how COVID-19 has changed reading habits.

The COVID-19 crisis forced Ukrainian bookstores and libraries to close across the country, which has ignited an online book-shopping. According to our survey conducted in February 2021, 74\% of respondents in Kyiv prefer printed books. During the quarantine, Kyiv bookstores acted as book delivery points. Readers still preferred to order books offline, in book delivery points.

Second, Ukrainian libraries are still unpopular. Right now, COVID-19 is the biggest challenge facing libraries today, but other problems await too. New tools of information technology have absolutely changed the role of library. But the Ukrainian government is not giving «desired priority» to their library industry while allocating funds.

Third, most part of readers chose Russian-language books. Also, they prefer foreign authors to Ukrainian authors.

\section{REFERENCES}

[1] Bukva. (2021). About Company. Retrieved from https://bukva.ua/en/html/about_company.

[2] Ivan Fedorov Book Chamber of Ukraine. (2021). Book publishing in Ukraine. Retrieved from http://www.ukrbook.net/statistika_html.

[3] The Book Platform. (2012). Publishing and Bookselling in Ukraine. Retrieved from http://www.bookplatform.org/en/activities/47publishing-ukr-en.html.

[4] Gutenbergz, Cultural and publishing project Chytomo, Razumkov Centre. (2018). Ukrainian Reading and Publishing Data. Retrieved from http://data.chytomo.com/en/knyzhka-za-tsinoyu-obidu-skilkyukrayintsi-gotovi-platyty-za-chytannya-ukrayintsi- $2 /$.

[5] Shercliff, E. (2021). Publishing in Ukraine: a review of the sector. Final report 2020. British Council. Retrieved from https://www.britishcouncil.org.ua/sites/default/files/333_research_en_ gb_v10.pdf.

[6] Gerden, E. (2018). Retail Pressures: Ukraine's Open-Air Bookselling Marketplaces Appear Headed for Closure. Publishing Perspectives. Retrieved from https://publishingperspectives.com/2018/07/retailpressures-ukraine-open-air-bookselling-marketplaces/.
[7] Stepurin, I. (2019). Book market of Ukraine. Sammit Books . Retrieved from http://www.library.univ.kiev.ua/ukr/for_lib/konf-20191/stepuryn.pdf.

[8] Gerden, E. (2020). Ukraine Book Business After Lockdowns: An 'Almost Complete Collapse. Publishing Perspectives. Retrieved from https://publishingperspectives.com/2020/08/ukraine-industryfollowing-lockdown-an-almost-complete-collapse-covid19/.

[9] Rybak, V. (2020). Reading in Ukrainian: The Resurgence of the Ukrainian Book Industry. Ukraine World International. Retrieved from https://ukraineworld.org/articles/ukraine-explained/reading-ukrainianresurgence-ukrainian-book-industry..

[10] Lavina mall. (2021). Bukva. Retrieved from https://lavinamall.ua/en/establishments/bukva/e76.

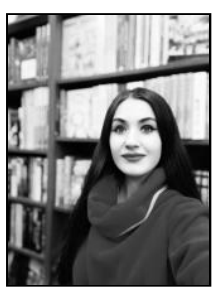

Khrystyna Astaptseva is a young (born 07 April 1991) Ukrainian researcher of women's press, feminism and fashion journalism. The first higher education of a Fashion designer (Lviv Professional College of Fashion Industry, 2010; Khmelnytskyi National University, 2013) and the second higher education of an Editor \& Publisher (Ukrainian Academy of Printing, 2016), as well as PhD-studies in journalism (Ukrainian Academy of Printing, 2020) helped Khrystyna to research women's magazines and fashion publications. Working in the State Scientific Institution "Book Chamber of Ukraine named after Ivan Fedorov" and the National Library of Ukraine named after V.I. Vernadsky (VNLU), Khrystyna Astaptseva significantly deepened the bibliographic, bibliological and publishing knowledge of the researcher.

Khrystyna Astaptseva is a follower of well-known Ukrainian Media researchers: Prof. Dr. Lidiya Snitsarchuk and PhD Valentina Perediriy. The result of an in-depth study of the Galician women's press was the discovery of the names of a cohort of Ukrainian fashion commentators of the 1930s: Kharytyna Kononenko, Stefania Martyniuk, Emanuila Olesnytska-Novak, Mika Drohomyretska, Mika Zelena-Bachynska and many others.

Together with the supervisor, prof. Nadiya Zelinska, Khrystyna developed and implemented the concept of the first Ukrainian bibliographic index of publications about fashion in the women's press (late XIX - late third of the XX century), which also introduces the reader to the original texts of Lviv fashion journalists, the most important in terms of Ukrainian fashion history. During 2016-2021 Khrystyna already published 20 manuscripts, from which $20 \%$ have international published character.

Publications in ukrainian:

https://chtyvo.org.ua/authors/Astaptseva_Khrystyna/.

Publications in english:

https://www.journalcra.com/sites/default/files/issue-pdf/39242.pdf

URL: http://www.irbis-nbuv.gov.ua/everlib/person/070475.

ORCID iD: https://orcid.org/0000-0003-1578-5747. 